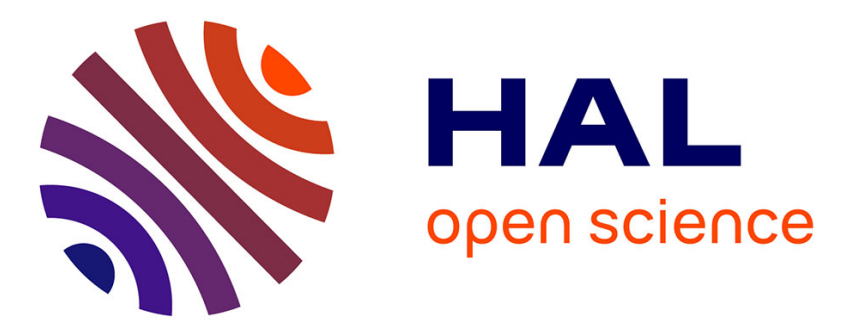

\title{
Inactivation de deux virus pathogènes pour les salmonidés (virus de la nécrose pancréatique infectieuse et de la septicémie hémorragique virale) par les rayons ultraviolets
}

Gérard Maisse, M. Dorson, C. Torchy

\section{To cite this version:}

Gérard Maisse, M. Dorson, C. Torchy. Inactivation de deux virus pathogènes pour les salmonidés (virus de la nécrose pancréatique infectieuse et de la septicémie hémorragique virale) par les rayons ultraviolets. Bulletin francais de Pisciculture, 1980, 278, pp.34-40. hal-01600820

\section{HAL Id: hal-01600820 \\ https://hal.science/hal-01600820}

Submitted on 2 Jun 2020

HAL is a multi-disciplinary open access archive for the deposit and dissemination of scientific research documents, whether they are published or not. The documents may come from teaching and research institutions in France or abroad, or from public or private research centers.
L'archive ouverte pluridisciplinaire HAL, est destinée au dépôt et à la diffusion de documents scientifiques de niveau recherche, publiés ou non, émanant des établissements d'enseignement et de recherche français ou étrangers, des laboratoires publics ou privés.

\section{다(1)(2)}

Distributed under a Creative Commons Attribution - ShareAlikel 4.0 International 


\section{BULLETIN FRANÇAIS DE PISCICULTURE}

\section{INACTIVATION \\ DE DEUX VIRUS PATHOGÈNES \\ POUR LES SALMONIDES (VIRUS DE LA NÉCROSE}

PANCRÉATIQUE INFECTIEUSE ET DE LA SEPTICÉMIE HÉMORRAGIQUE VIRALE) PAR LES RAYONS ULTRAVIOLETS

G. MAISSE *, M. DORSON **, C. TORCHY**

RESUME

Le virus de la Nécrose Pancréatique Infectieuse (N.P.I.) et le virus de la Septicémie Hémorragique Virale (S.H.V.) ont été dilués dans de l'eau claire, puis inactivés par passage à différents débits dans un appareil U.V., fabriqué au Laboratoire.

Le titrage d'échantillons de l'effluent a permis le calcul d'une D.L. 90 de $10000 \mu \mathrm{Ws} \mathrm{cm}^{-2}$ pour le virus de la S.H.V. Le virus de la N.P.I. a montré une plus grande résistance, avec une D.L. 80 de $330000 \mu \mathrm{Ws} \mathrm{cm}$.

Dans les conditions de la Salmoniculture, la protection des écloseries à l'aide des U.V. contre les virus européens des Salmonidés ne peut être pratiquée que dans le cas du virus de la S.H.V. et non contre le virus de la N.P.I.

* Laboratoire de Physiologie des Poissons, I.N.R.A., RENNES.

** Laboratoire d'Ichtyopathologie, I.N.R.A., THIVERVAL-GRIGNON. 


\section{SUMMARY}

\section{ULTRAVIOLET INACTIVATION OF TWO PATHOGENIC SALMONID VIRUSES (I.P.N. Virus and V.H.S. Virus).}

Infectious Pancreatic Necrosis Virus (I.P.N.V.) and Viral Haemorrhagic Septicaemia Virus (V.H.S.V.) were diluted in clear tap water and subsequently inactivated by passing through a laboratory made U.V. system at various flow rates.

Titration of outflowing samples allowed calculation of a L.D. 90 of $10000 \mu$. W.s. $\mathrm{cm}^{-2}$ for V.H.S.V.. I.P.N.V. proved much more resistant, with a L.D. 80 of $330000 \mu$.W.s. $\mathrm{cm}^{-2}$.

Under trout farming conditions, U.V. protection of hatcheries against european Salmonid viruses could be achieved only in the case of V.H.S.V. and not against I.P.N.V.

\section{INTRODUCTION}

L'importance des maladies virales de la Truite arc-en-ciel (Salmo gairóneri) et l'absence de traitement disponible nous ont amenés à tester l'efficacité des rayons ultraviolets (U.V.) pour la désinfection d'une eau contaminée soit par le virus de la Septicémie Hémorragique Virale (S.H.V.) (ZWILLENBERG et al., 1965), soit par le Virus de la Nécrose Pancréatique Infectieuse (N.P.I.) (WOLF et al., 1960).

$\mathrm{Si}$ les rayonnements U.V. sont couramment utilisés dans le traitement des eaux potables, pour la lutte contre les bactéries et les virus, leur utilisation en Ichtyopathologie est surtout limitée à l'aquariophilie.

L'effet germicide maximum est produit par des longueurs d'onde comprises entre 2500 et 2600 A dont l'absorption est affectée principalement par la turbidité, les composés organiques et la teneur en sels ferreux. (HUFF et al. 1965).

Divers dispositifs sont disponibles dans le commerce, mais les travaux rapportés ici ont été réalisés à l'aide d'un système fabriqué, à l'exception de la lampe, au Laboratoire, permettant de masquer une partie de la lampe.

\section{MATERIEL ET METHODE}

\section{1) Dispositif expérimental :}

Le dispositif utilisé (fig. 1) fait appel à une unité de recyclage (pompe et bac 1) de laquelle l'eau peut être dérivée sous un débit connu vers un bac de décharge 2 en passant sous pression par le tube générateur de rayons U.V.

Nous avons utilisé une lampe "MAZDA GERMICIDE TG 30 ", placée dans un tube en PVC de diamètre intérieur $9,8 \mathrm{~cm}$ dans lequel la circulation de l'eau était ascendante. La longueur de stérilisation était réduite à $60 \mathrm{~cm}$.

Durant les différentes expérimentations, la température de l'eau était comprise entre $10^{\circ} \mathrm{C}$ et $12^{\circ} \mathrm{C}$ et le pH égal à 7,3 , la teneur en fer ferreux était inférieure à $0,01 \mathrm{mg} / 1$.

L'intensité germicide U.V. est donnée par la formule :

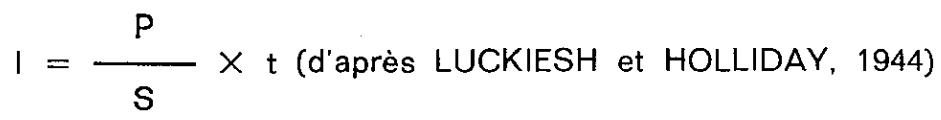

où $\mathrm{P}$ est la puissance totale émise à $2537 \AA$ (ici $9000 \mu \mathrm{W}$ )

$S$ est la surface de la lampe (ici $698,6 \mathrm{~cm}^{2}$ )

$t$ est le temps de passage d'une particule devant la lampe (en sec.).

La valeur de I, donnée en microwatt seconde par centimètre carré $\left(\mu \mathrm{Ws} / \mathrm{cm}^{2}\right)$ pouvait être fixée en modifiant le débit de l'effluent (de $600 \mathrm{l} / \mathrm{h}$ à $3200 \mathrm{l} / \mathrm{h}$ ) ou (et) en masquant une partie de la lampe à l'aide d'un tube opaque. 


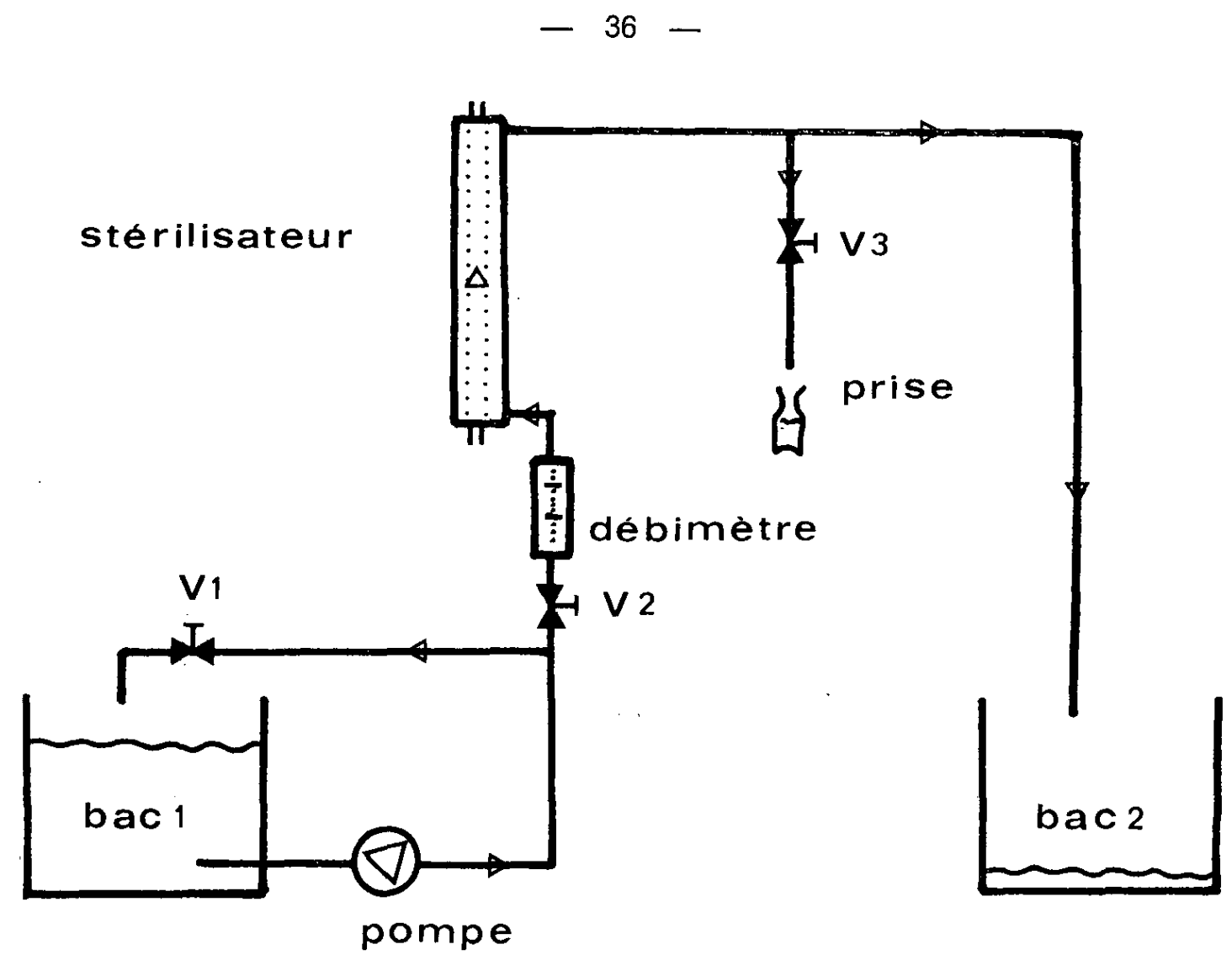

Figure 1 : Schéma de l'installation utilisée pour les essais d'inactivation des Virus de la S.H.V. et de la N.P.I. par les U.V.

Environ $3 \times 10^{9}$ unités formant plage (ufp) du virus étaient dispersées dans 3001 d'eau contenus dans le bac 1 , la vanne $V 2$ étant fermée et $V 1$ ouverte, la pompe fournissant alors un débit de recyclage de $3200 \mathrm{l} / \mathrm{h}$.

Au bout de $10 \mathrm{mn}$ et $20 \mathrm{mn}$, deux prises d'essai en $\mathrm{V} 1$ permettaient de contrôler la bonne dispersion des particules virales.

Le débit désiré entre le bac 1 et le bac 2 était obtenu par réglage des vannes $V 1$ et $\vee 2$. La prise d'échantillon à titrer était pratiquée en $V 3$ après purge de 1 litre environ. Une prise de contrôle final était effectuée de la même façon en V 3 après extinction de la lampe.

Entre deux expérimentations, l'installation était désinfectée aux iodophores (GERARD, 1974), qui étaient ensuite neutralisés puis éliminés par rinçage abondant.

2) Les virus et leur dénombrement dans les échantillons : (GRIGNON).

Le virus de la S.H.V. (virus d'EGTVED) utilisé est la souche pathogène 07/71

Le virus de la N.P.I. est la souche pathogène 31/75 (GRIGNON).

Après dilution du prélèvement au 1/10 en milieu de Eagle, les deux virus sont titrés par la méthode des plages sous agarose classiquement utilisée (DE KINKELIN et SCHERRER, 1970). Des monocouches de cellules de Truite $R_{T} G_{2}$ en boite de pétri de $35 \mathrm{~mm}$ reçoivent $0,1 \mathrm{ml}$ de la suspension virale et sont recouvertes $1 \mathrm{~h}$ plus tard de milieu nutritif gélosé à $0,5 \%$. Après incubation $\left(3\right.$ jours à $14^{\circ} \mathrm{C}$ ) et coloration des cellules au Rouge Neutre, les plages de lyse (correspondant chacune à une unité virale dans la suspension de départ) 
sont dénombrées. Le pourcentage d'inactivation est donné par : $P=\left(1-\frac{N}{\text { No }}\right) \times 100$ ou No = nombre initial de virions et $N=$ nombre de virions après passage dans le stérilisateur.

\section{RESULTATS ET DISCUSSION}

Les résultats de l'inactivation des deux virus ont été reportés sur des courbes du type : (figure 2)

$$
\begin{aligned}
\mathrm{P} & =\left(1-\mathrm{e}^{-\mathrm{KI}}\right) \times 100 \\
\text { où } \quad \mathrm{P} & =\text { pourcentage d'inactivation } \\
\mathrm{I} & =\text { intensité germicide } \\
\mathrm{K} & =\text { constante. }
\end{aligned}
$$

Ceci conformément aux résultats de LUCKIESH et HOLLIDAY (1944) qui donnent la relation entre le taux de survie de la bactérie Escherichia coll et l'intensité germicide :

$$
\begin{aligned}
& \frac{N}{N o}=e^{-E t / Q} \\
& N=\text { Nombre de survivantes } \\
& \text { No }=\text { Nombre initial } \\
& E=\text { Intensité du rayon } \\
& t=\text { Temps d'exposition de la particule } \\
& \mathrm{Q}=\text { Dose mortelle. }
\end{aligned}
$$

Les pourcentages d'inactivation de $100 \%$ rapportés dans cette étude sont dus à la méthode de calcul à partir d'un échantillon. D'après les formules mathématiques, les $100 \%$ représentent l'asymptote vers laquelle tendent les courbes.

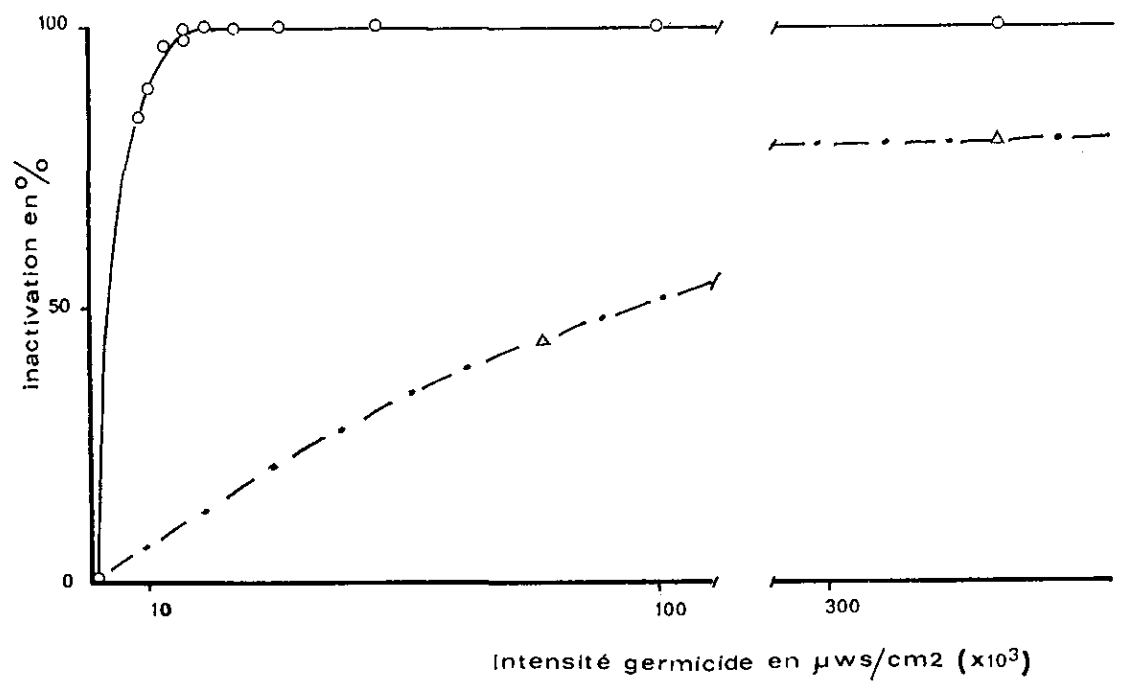

Figure 2 : Inactivation des virus de la S.H.V. $(0-0)$ et de la N.P.I. $(\Lambda-.-\Lambda)$ par les rayons U.V. Les résultats sont exprimés en pourcentage d'inactivation par rapport aux prélèvements témoins. 
Une très nette différence de sensibilité aux U.V. apparait entre les virus de la SHV et de la NPI.

Nous constatons une DL90 de $10000 \mu \mathrm{Ws} / \mathrm{cm}^{2}$ pour le virus de la S.H.V. et une DL 80 de $330000 \mu \mathrm{Ws} / \mathrm{cm}^{2}$ pour le virus de la N.P.I. Dans ce dernier cas, nos installations ne nous ont pas permis de déterminer une DL 90.

La figure $n^{\circ} 3$ replace les virus de la S.H.V. et de la N.P.I. par rapport à d'autres organismes pathogènes des Salmonidés, du point de vue de leur inactivation par les rayons U.V. en eau claire.

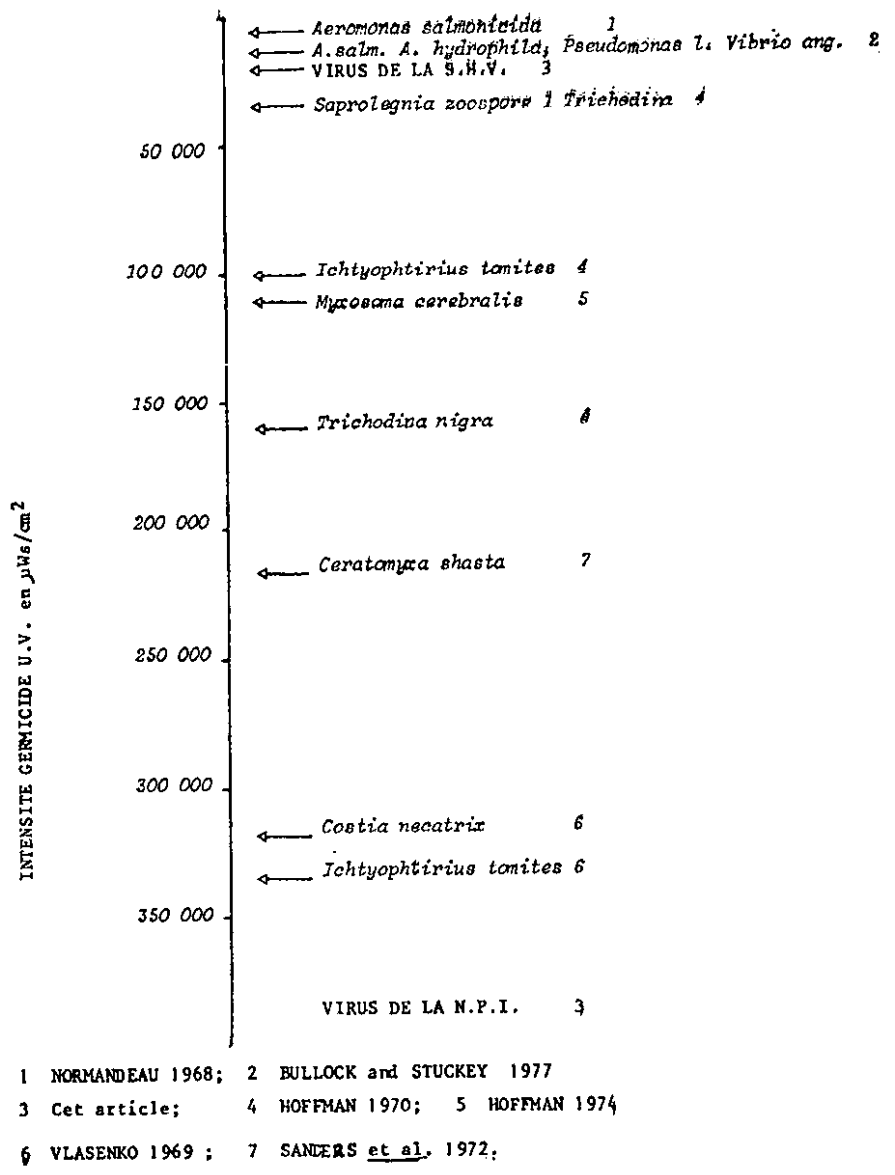

Figure $3^{\prime}$ : Comparaison des intensités germicides U.V. nécessaires à la destruction de différents agents ichtyopathogènes.

Si la N.P.I. apparait comme l'agent ichtyopathogène testé le plus résistant, il est intéressant de constater que le virus de la S.H.V. se situe parmi ceux qui sont les plus sensibles avec Aeromonas salmonicida, Aeromonas hydrophila, Pseudomonas fluorescens, Vibrio augnillarum et les zoospores de Saprolegnia sp pour qui les doses léthales sont toutes inférieures à $50000 \mu \mathrm{Ws} / \mathrm{cm}^{2}$.

Ces résultats concernent des eaux de turbidité voisine de zéro. Leur application en pisciculture doit être réservée à des eaux très claires, ou préalablement filtrées. C'est ainsi que BULLOCK et STUCKEY (1977) ont montré que l'on pouvait empêcher la transmission expérimentale de la furonculose en filtrant puis en irradiant par les U.V. l'eau ayant hébergé des Truites fario malades, avant de servir à infecter de jeunes Saumons atlantiques. 


\section{CONCLUSION}

La désinfection des eaux de pisciculture par les U.V. ne peut raisonnablement être envisagée que pour les agents pathogènes sensibles à des intensités germicides inférieures à $50000 \mu \mathrm{Ws} / \mathrm{cm}^{2}$, pour d'évidentes raisons de coût.

L'utilisation des rayonnements U.V. peut être un moyen de couper la transmission de la S.H.V., le virus de la N.P.I. étant trop résistant.

La grande résistance du virus de la N.P.I. aux rayons U.V. avait déjà été remarquée (MACKELVIE et DESAUTELS 1975, DOORSON, résultats non publiés), dans des conditions différentes (inactivation d'une suspension virale en couche mince dans une boite de pétri placée sous une lampe germicide).

Le dimensionnement des stérilisateurs devra tenir compte du vieillissement des lampes. Le flux énergétique de la lampe Mazda TG 30 tombe à $80 \%$ au bout de 2000 heures et $75 \%$ au bout de 5000 heures (données du constructeur).

L'utilisation des U.V. devra être réservée à des eaux très claires ou filtrées et, toujours compte tenu des coûts, on se limitera aux postes nécessitant peu d'eau : incubation, premier alevinage et recyclage.

Dans ce dernier cas elle peut se révéler utile à la fois sur l'alimentation en eau nouve (introduction d'agents pathogènes) et sur le débit recyclé pour limiter le nombre de germes dans le circuit.

La désinfection des eaux de pisciculture par les rayons U.V. peut, dans les conditions précédemment décrites, être retenue comme moyen de lutte contre notamment la furonculose, la S.H.V., et les mycoses.

\section{REFERENCES BIBLIOGRAPHIQQUES}

BULLOCK G.L., and H.M. STUCKEY, 1977. Ultraviolet treatment of water for destruction of five gram - negative bacteria pathogenic to fishes. J. Fish. Res. Board. Can. $34,(8)$.

DE KINKELIN P., et R. SCHERRER, 1970. Le Virus d'Egtved. I. - Développement, stabilité et structure de la souche danoise. Ann. Rech. Veter. I (1).

GERARD J.P., 1974. Note technique $n^{\circ} 03$ sur l'emploi des iodophores en pisciculture. Bull. Franç. Pisc. (254).

HOFFMAN G.I., 1970. Cité par HOFFMAN G.I. 1974.

HOFFMAN G.I., 1974. Désinfection of contamined water by ultraviolet irradiation, with emphasis on whirling disease (Myxosoma cerebralis) and its effect on fish. Trans. Amer. Fish. Soc. (3).

HUFF C.B., SMITH H.F., BORING W.D. and CLARKE N.A., 1965. Study of ultraviolet desinfection of water and factors in treatment efficiency. Public Health Rep. 80, (8).

LUCKIESH M., and HOLLIDAY L.L., 1944. Desinfecting water by means of germicidal lamps. General electric Rev. 47.

Mac KELVIE R.M., DESAUTELS D., 1975. Fish viruses : Survival and inactivation of infectious Pancreatic Necrosis Virus. J. Fish. Res. Board. Can. 32, (8).

NORMANDEAU D.A., 1968. Progress Report, Project F14 R3. State of New Hampshire (Mimeo). 
SANDERS J.E., FRYER J.L., LEITH D.A., and MOORE K.D., 1972. Control of the infectious protozoan Ceratomyxia shasta by treatment of hatchery water supplies. Prog. Fish Cult. 34 (1).

VLASENKO M.I., 1969. Ultraviolet rays as a method for the control of diseases of fish eggs and young fishes. Problems of Ichtyol. 9.

WOLF K., SNIESZKO S.F., DUNBAR C.E., and PYLE E.A. 1960. Virus nature of infectious Pancreatic Necrosis in trout. Proceeding of the Society for Experimental Biology and Medicine. 104.

ZWILLENBERG L.D., JENSEN M.H., and ZWILLENBERG H.L., 1965. Electron microscopy of the virus of Viral Haemornhagic Septicemia of rainbow Trout. Archiv. für die gesamte Virus forschung, 17. 